\title{
Estimating Solar Insolation and Power Generation of Photovoltaic Systems Using Previous Day Weather Data
}

\author{
Min Hee Chung \\ School of Architecture and Building Science, Chung-Ang University, Seoul 06974, Republic of Korea \\ Correspondence should be addressed to Min Hee Chung; mhloveu@cau.ac.kr
}

Received 22 October 2019; Revised 2 January 2020; Accepted 16 January 2020; Published 18 February 2020

Academic Editor: Emanuele Brunesi

Copyright ( $\odot 2020$ Min Hee Chung. This is an open access article distributed under the Creative Commons Attribution License, which permits unrestricted use, distribution, and reproduction in any medium, provided the original work is properly cited.

\begin{abstract}
Day-ahead predictions of solar insolation are useful for forecasting the energy production of photovoltaic (PV) systems attached to buildings, and accurate forecasts are essential for operational efficiency and trading markets. In this study, a multilayer feedforward neural network-based model that predicts the next day's solar insolation by taking into consideration the weather conditions of the present day was proposed. The proposed insolation model was employed to estimate the energy production of a real PV system located in South Korea. Validation research was performed by comparing the model's estimated energy production with the measured energy production data collected during the PV system operation. The accuracy indices for the optimal model, which included the root mean squared error, mean bias error, and mean absolute error, were $1.43 \mathrm{kWh} / \mathrm{m}^{2} / \mathrm{day},-0.09 \mathrm{kWh} / \mathrm{m}^{2} /$ day, and $1.15 \mathrm{kWh} / \mathrm{m}^{2} /$ day, respectively. These values indicate that the proposed model is capable of producing reasonable insolation predictions; however, additional work is needed to achieve accurate estimates for energy trading.
\end{abstract}

\section{Introduction}

Electricity consumption has been rapidly increasing around the world despite efforts to improve energy conservation. In 2013, in Korea, the electricity consumption in the commercial and public sectors increased by $10 \%$ to $65.8 \%$ compared to values in 2001. Moreover, the electrification of energy consumption has further intensified [1]. Since natural gas and electricity have emerged as primary energy sources in the household sector, this share of electricity consumption has been steadily increasing. This is because households prefer clean energy sources to maintain comfort and the convenience of living. The Korean government is promoting buildings in which renewable energy systems are used, to save on primary energy consumption and to realize energy independence.

Solar energy is known to be a good substitute for fossil fuels, which currently account for more than $80 \%$ of the primary energy supply [2]. Photovoltaic (PV) systems are the most suitable replacements for fossil fuels because they do not produce $\mathrm{CO}_{2}$ emissions and do not pose the same risks as those associated with other alternative energy supplies such as nuclear power generation. It may be more desirable to install
PV systems in the city rather than in the rural or forested areas because of land conservation concerns. Indeed, PV systems are more likely than other types of renewable energy systems, to be constructed in urban built environments.

The Korean government intends to introduce a smart grid to promote the embracement of renewable energy [3]. As part of these efforts, the government announced a second basic plan for an intelligent power grid in 2018. Accordingly, the government has set up policies on smart grid complex construction, infrastructure, and facility expansion. It was reported that, in 2019, a distributed energy resource market would be allowed in Korea. As a result, significant changes are expected in the electric trading market, which has been focused on centralized power generation companies. The expansion of distributed generation will accelerate direct energy trading among individuals and groups that can produce and consume energy simultaneously $[3,4]$. Such peer-to-peer (P2P) energy trading has been demonstrated in various forms in the United States, United Kingdom, Netherlands, and Germany [5-7]. P2P energy trading is beneficial because it reduces the need for expensive and inefficient energy transportation with substantial losses [8], 
thus improving the reliability of energy supply [9] and alleviating transmission and distribution congestion problems [10]. Forecasting solar insolation in advance is essential in the management of the PV system's generated output in a distributed grid. It is necessary to provide information on the energy production through the system to the prosumer, who conducts the small-scale electricity trading as a weather forecast in advance. Time-based predictions may need to be provided according to the individual's characteristics. However, it is necessary to provide daily production forecast information to a large number of prosumers. In small-scale energy trading, the provision of daily energy production forecast information is more effective than providing detailed hourly energy generation forecast information. Daily energy production by PV systems can be predicted simply based on daily irradiation.

Many researchers are presently directing their efforts on solar insolation forecasting techniques. Soares et al. [11] examined the intrahour solar insolation predictions based on hourly weather data for a period of 4 years. They estimated the hourly values of the diffuse solar radiation by using multilayer perceptron neural networks. However, their model could not reflect the present-day weather change. Mathiesen and Kleissl [12] compared five forecasting models for the intraday solar insolation by using ground-based weather measurement data, and these efforts resulted in the production of an accurate database to validate numerical weather predictions. The accuracy of each model in predicting insolation varied in accordance with the weather conditions. However, it was difficult to consider multiple models simultaneously. Sun et al. [13] proposed a random forest algorithm for estimating the daily solar radiation based on meteorological data, solar radiation, and three air pollution indexes for $\mathrm{SO}_{2}, \mathrm{NO}_{2}$, and PM10. They optimized the proposed estimation model depending on the input variables. In a city with a high degree of air pollution, this model can be used to estimate the exact amount of solar radiation; however, the number of input variables increases. Sharma and Kakkar [14] also proposed an hourly global solar irradiance model for different forecasting horizons ranging from a few hours ahead to 48 hours ahead by using machine learning. The model with 1 hour ahead predictions was the most accurate; however, there was not enough time to provide information to users. Gutierrez-Corea et al. [15] modeled the spatial-temporal short-term global solar insolation by using artificial neural networks. This model gave accurate data predictions over time but increased the complexity of the prediction model with more than 900 input parameters. Huang and Davy [16] proposed a linear regression model for the intrahour solar irradiance that uses the hourly clear sky index and geopotential thickness. The model predicted hourly solar irradiance only for summer. Therefore, there were limitations in applying this model to other seasons. Vakili et al. [17] designed a prediction model for the total daily solar insolation for Iran by using a multilayer perceptron artificial neural network. The limitation of this model was that the solar insolation was estimated based on the weather observation information, rather than the future value. Qing and Niu [18] presented a novel solar irradiance prediction method for hourly day-ahead forecasts by using hourly weather forecast data. The proposed model contained structured long- and short-term memory networks, and it was compared with other algorithms. By using weather forecasting data as input variables, it is possible to reflect the change in solar radiation in accordance with the weather changes. However, the accuracy of the solar radiation prediction changes based on the prediction accuracy of the weather forecast data. Amrouche and Le Pivert [19] proposed a solar radiation forecasting model using artificial neural networks (ANNs) and special modelling. In cases where there were no meteorological data from the predicted area, the solar radiation was estimated using meteorological data from the nearby areas. This model predicted daily values, which were used to estimate power generation by PV systems. Long et al. [20] proposed a prediction model for the daily PV energy production by meteorological parameters. The efficiency of the prediction algorithm was improved by classifying the weather data based on importance and with reduced input variables used as input data.

The prediction accuracy varied in accordance with the accuracy of the weather forecasts. In addition, there have been many studies on various solar insolation prediction models with different prediction horizons and different prediction methods. The previous solar forecasting models made predictions based on the time or day. However, a large amount of input data was needed for accuracy within the predicted time scale. The limitation of the prediction model is that the training and prediction time increases as the number of input variables increases. This study aims to develop a simple solar insolation prediction model by using fewer weather data variables, which make it easy to acquire information. Typically, weather forecast data may be used to predict the next day's solar insolation. However, predicting the next day's solar insolation using weather forecast data is characterized by a lot of uncertainty due to the uncertainty in the weather forecast data.

The objective of this paper is to propose a simple prediction model for day-ahead solar insolation using weather observation data. The predicted solar insolation would be used for estimating energy production in advance. The estimated energy production would give information for the determination of optimal operating conditions, such as selfconsumption or feed-in into the grid mode for PV systems located in a distributed grid. The day-ahead solar insolation prediction model forecasts the solar radiation of the next day based on the weather data of the current day. It is assumed that the predicted model cannot be applied to systems installed in a specific condition; however, it can predict information applicable to a wide range of conditions. Therefore, the weather difference due to the differences in the meteorological observation site and the installation location of the PV system was ignored. The input variables use meteorological data that can be easily obtained from the meteorological administration. The prediction model could provide building users with useful information to plan their energy use in advance. This paper is organized as follows. In Section 2, the data used for training and checking the 
prediction model are described. Section 3 explains the prediction model and the verification method. The measured energy production for a PV system located in South Korea was compared to the energy production prediction obtained through the solar insolation prediction model. Section 4 presents the results, and Section 5 presents the conclusions.

\section{Meteorological Data}

The meteorological data used in this study were provided by the Korea Meteorological Administration. Ground observations were carried out by manual and automatic observations for the automated synoptic observing system. The temperature, humidity, wind direction, wind velocity, air pressure, precipitation, sunshine, solar radiation, surface temperature, grass temperature, and ground temperature were automatically obtained by the use of synoptic weather observation equipment, which report atmospheric conditions near the ground in real-time. Snowfall, clouds, and other daily phenomena were observed manually every hour or every three hours.

The air temperature, which varied with the measured height, was measured at a height of about 1.2 to $1.5 \mathrm{~m}$ above the ground. The wind direction was represented by a vector quantity with a direction and magnitude. However, since the horizontal component was much larger than the vertical component, generally only the horizontal component was observed. Air flow at a height of $10 \mathrm{~m}$ from the ground was also observed. Precipitation refers to rain, snow, and hail, i.e., liquid and solid precipitation. In the case of solid precipitation, the depth of the precipitation was measured. Solar insolation was automatically observed by a pyranometer every hour. Data on sunshine hours and sunshine duration were collected. These data were then used to compute the continued sunshine duration, which is a measure of the duration of continuous reflection of sunshine on the ground surface without blockage by clouds or fog. Clouds were manually observed in terms of their shape, height, and cover. Other meteorological phenomena such as the visibility, ground surface, minimum grass surface, and ground temperatures were observed. The solar insolation was determined by the Sun's altitude and azimuth with respect to the season and time. However, the solar insolation reaching the Earth's surface varied depending on the atmospheric weather conditions.

The weather data for the prediction model were recorded in Seoul, South Korea, and the station specifications are given in Table 1. The dataset collection period lasted for 4 years (2014-2017). Data that had some elements missing were excluded from the dataset. The dataset was divided into training and testing datasets. For the training dataset, data ranging from 2014 to 2016 were used. For testing, the 2017 data were used. The total training and testing datasets consisted of 1084 and 357 days, respectively. The variables were selected based on the results of a previous study [21], which analyzed the correlation between solar insolation and weather data. Temperature, humidity, precipitation, precipitation duration, wind speed, sunshine duration, continued sunshine duration, and cloud cover were selected as
TABLE 1: Station specifications.

\begin{tabular}{lc}
\hline Item & Specification \\
\hline Location & Seoul, South Korea \\
Latitude & $37.5714^{\circ} \mathrm{N}$ \\
Longitude & $126.9658^{\circ} \mathrm{E}$ \\
Elevation & $85.67 \mathrm{~m}$ \\
Climate type & Humid continental \\
Observed period & $2014-2017$ \\
\hline
\end{tabular}

initial input variables that could affect the solar radiation, as depicted in Table 2. Visibility can especially be influenced by aerosols in the air. According to Chung [22], there is a low correlation between the ratio of the horizontal global radiation to the extraterrestrial radiation and particulate matter in Korea. In this study, the effects of wind direction, cloud shapes, heights of clouds, visibility, ground temperatures, and surface ground temperature were excluded.

In the next step, all the data were normalized to a scale between zero and one based on equation (1); normalization of the input reduces estimation errors and makes learning fast and efficient [23]:

$$
y_{i}=\frac{x_{i}-\operatorname{MIN}(x)}{\operatorname{MAX}(x)-\operatorname{MIN}(x)},
$$

where $x_{i}$ is the observed data at time $i, y_{i}$ is the normalized data at time $i$, and $\operatorname{MIN}(x)$ and $\operatorname{MAX}(x)$ are the minimum and maximum values during the observation period, respectively.

\section{Model Methods and Evaluation Indices}

A forecast model based on the weather data is presented in this section, along with the model verification procedures. The solar insolation presented through the predictive model is the value of the horizontal plane insolation; therefore, the insolation on the inclined PV module is calculated. This solar insolation of the inclined plane will be used to calculate the energy output of the PV system and compare it with the energy output of the PV system being monitored. Finally, the error verification method used in this paper is presented.

3.1. Forecast Method. This study used a type of artificial neural network (ANN) architecture known as a multilayer feed-forward neural network (MLF) for the modelling. The multilayer feed-forward neural network is also referred to as a multilayer perceptron (MLP). This model is similar to the persistence model in that the previous day's data are used as the input variables. The persistence model assumes that the conditions are unchanged between the current time and the future time [24]. However, because the sun's position and weather conditions change from day to day, it cannot be assumed that data on weather conditions are the same as those of the previous day. In particular, it is difficult to apply a persistence model in the case of a 1-day forecast horizon. For ANN models, the magnitudes of weights and biases are changed in a time series, which makes them more appropriate [25]. Diagne et al. suggested an appropriate model by 
TABLE 2: The weather variables at initial time.

\begin{tabular}{lcc}
\hline Variables & Min & Max \\
\hline Lowest temperature $\left(T_{\text {low }},{ }^{\circ} \mathrm{C}\right)$ & -18.0 & 28.7 \\
Highest temperature $\left(T_{\text {high }},{ }^{\circ} \mathrm{C}\right)$ & -10.5 & 36.6 \\
Precipitation $(\mathrm{PR}, \mathrm{mm})$ & 0 & 144.5 \\
Precipitation duration $(\mathrm{PD}, \mathrm{hr})$ & 0 & 24 \\
Minimum humidity $(\mathrm{RH}, \%)$ & 7 & 94 \\
Daily wind speed (DWS, m/s) & 0.9 & 6.4 \\
Sunshine duration $(\mathrm{SD}, \mathrm{hr})$ & 9.6 & 14.8 \\
Continued sunshine duration $(\mathrm{CSD}, \mathrm{hr})$ & 0 & 13.7 \\
Cloud cover $(\mathrm{CC},-)$ & 0 & 10 \\
\hline
\end{tabular}

forecasting the horizon [26]. The persistence model is appropriate when the forecasting horizon is within an hour, but the ANN model is suitable when the forecasting horizon is longer than an hour. Trained with a backpropagation learning algorithm, MLF is one of the most popular types of ANN architectures used to forecast solar insolation $[15,27-30]$. The MLF was implemented in MATLAB. The MLF consists of activation functions, bias, and neurons [31]. The neurons were ordered into input and hidden and output layers, as shown in Figure 1. The number of hidden layers (NHLs) was set to be between 1 and 5. This was done because the forecast model was supposed to forecast the output of nonlinear relationships between input data. Moreover, the model was complicated. The range of the number of hidden neurons was determined by the initial number of hidden neurons, which was calculated based on equation (2) by using the number of input neurons [32]. Each layer consists of the same number of neurons:

$$
\mathrm{NHN}=2 \mathrm{NIN}+1 \text {, }
$$

where NHN is the number of hidden neurons and NIN is the number of input neurons.

The Levenberg-Marquardt method for training was used in this study. The Levenberg-Marquardt method is the most representative method for solving nonlinear least squares problems $[33,34]$. The sliding-window method that provides a higher accuracy was used for controlling the training dataset $[32,35,36]$.

The initial input variables were obtained by predicting the solar radiation using the nine input variables presented in the previous study and then refining the predicted values by adjusting the number of input variables. This prediction prevented a fitting problem by using normalized data and dropout method. The training parameters are as shown in Table 3.

3.2. Insolation on an Inclined Plane. The inclined insolation was calculated by Lid and Jordan's equation [37]:

$$
\begin{aligned}
K_{T}= & \frac{H}{H_{o}} \\
H_{\mathrm{o}}= & \frac{24}{\pi} \cdot I_{\mathrm{sc}}\left(1+0.033 \cos \frac{360 n}{365}\right) \\
& \cdot\left(\cos \phi \cos \delta \sin \omega_{\mathrm{s}}+\frac{\pi \omega_{\mathrm{s}}}{180} \sin \phi \sin \delta\right),
\end{aligned}
$$

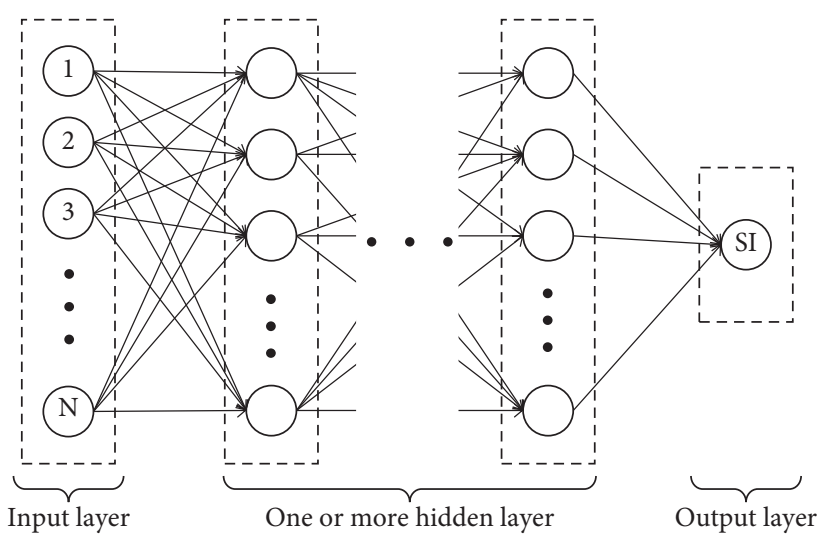

Figure 1: MLF architecture.

TABLE 3: Training parameters.

\begin{tabular}{lc}
\hline Parameters & Value \\
\hline Maximum number of epochs to train & 1000 \\
Performance goal & 0.01 \\
Minimum performance gradient & $1 e-7$ \\
Initial mu & 0.001 \\
Maximum mu & $1 e+10$ \\
Maximum validation failures & 6 \\
Epochs between displays & 50 \\
\hline
\end{tabular}

where $I_{\mathrm{sc}}$ is the solar constant $\left(1367 \mathrm{~W} / \mathrm{m}^{2}\right), n$ is the day number of year, $\phi$ is the latitude, $\delta$ is the solar declination, and $\omega_{\mathrm{s}}$ is the sunset hour angle. The values of $\delta$ and $\omega_{\mathrm{s}}$ can be approximately expressed by equations (4) and (5), respectively:

$$
\begin{aligned}
\delta & =23.45 \sin \left[\frac{360(n+284)}{365}\right], \\
\omega_{\mathrm{s}} & =\arccos (-\tan \delta \tan \phi) .
\end{aligned}
$$

The daily solar insolation on an inclined plane, $H_{t}$, can be expressed as

$$
H_{t}=R \cdot H
$$

where $R$ is defined to be the ratio of the daily mean insolation on an inclined plane to the mean insolation in a horizontal plane.

$$
R=\left(1-\frac{H_{\mathrm{d}}}{H}\right) R_{\mathrm{b}}+H_{\mathrm{d}}\left(\frac{1+\cos (s)}{2 H}\right)+\rho\left(\frac{1-\cos (s)}{d}\right),
$$

where $R_{\mathrm{b}}$ is the ratio of the average beam insolation on the inclined plane to that on a horizontal surface, $s$ is the tilted angle, and $\rho$ is the ground reflectance. $R_{\mathrm{b}}$ is a function of the atmosphere's transmittance and is calculated by

$$
R_{\mathrm{b}}=\frac{\cos (\phi-s) \cos (\delta) \sin \left(\omega_{\mathrm{s}}^{\prime}\right)+\omega_{\mathrm{s}}^{\prime}(\pi / 180) \sin (\phi-s) \sin (\delta)}{\cos (\phi) \cos (\delta) \sin \left(\omega_{\mathrm{s}}\right)+\omega_{\mathrm{s}}(\pi / 180) \sin (\phi) \sin (\delta)},
$$

where $\omega_{\mathrm{s}}^{\prime}$ is the sunset hour angle for the inclined plane and is estimated by equation (9). $H_{\mathrm{d}}$ is the daily diffuse solar insolation given by Page [38], as equation (10): 


$$
\begin{aligned}
\omega_{\mathrm{s}}^{\prime} & =\min \left\{\omega_{\mathrm{s}}, \operatorname{arccons}[-\tan (\phi-s) \tan (\delta)]\right\}, \\
H_{\mathrm{d}} & =H\left(1.00-1.13 K_{T}\right) .
\end{aligned}
$$

\subsection{Estimation Model and Monitoring the Photovoltaic} System Energy Production. For the evaluation of the applicability of the proposed solar radiation prediction model, the PV production obtained by using the estimated solar insolation was compared to the actual energy production. The PV system was assumed to be of the gridconnected type for the distributed generation. The generated energy was not stored in batteries. Energy production by the photovoltaic system was obtained by applying equations (11) and (12) [39-42]:

$$
\begin{aligned}
& E_{\mathrm{PV}}=A_{\mathrm{a}} \cdot H_{t} \cdot \eta_{\text {con }}, \\
& \eta_{\text {con }}=\eta_{\mathrm{STC}}\left(1-\beta\left(T_{\mathrm{c}}-T_{\mathrm{STC}}\right)\right) \cdot \eta_{\text {inv }}, \\
& T_{\mathrm{c}}=T_{\mathrm{a}}+\frac{\mathrm{SI}}{\mathrm{SI}_{\mathrm{NOCT}}}\left(T_{\mathrm{NOCT}}-T_{\mathrm{a} . \mathrm{NOCT}}\right),
\end{aligned}
$$

where $E_{\mathrm{PV}}$ is the energy delivered by photovoltaics (kWh), $A_{\mathrm{a}}$ is the area of the array $\left(\mathrm{m}^{2}\right), H_{t}$ is the insolation on the plane for the photovoltaics array $\left(\mathrm{kWh} / \mathrm{m}^{2}\right), \eta_{\text {con }}$ represents the conversion efficiency $(\%), \eta_{\text {STC }}$ is the module efficiency at STC (\%), $\beta$ is the temperature coefficient at the maximal power of the module $\left(\% /{ }^{\circ} \mathrm{C}\right), T_{\mathrm{c}}$ is the PV cell temperature $\left({ }^{\circ} \mathrm{C}\right), T_{\mathrm{STC}}$ is the standard test condition temperature $\left(25^{\circ} \mathrm{C}\right)$, $\eta_{\text {inv }}$ is the inverter efficiency (\%), $T_{\mathrm{a}}$ is the ambient temperature $\left({ }^{\circ} \mathrm{C}\right), \mathrm{SI}$ is the incident solar irradiance on the PV plane $\mathrm{PV}\left(\mathrm{W} / \mathrm{m}^{2}\right), \mathrm{SI}_{\mathrm{NOCT}}$ is the incident solar irradiance of $800 \mathrm{~W} / \mathrm{m}^{2}, T_{\text {NOCT }}$ is the nominal operating cell temperature $\left({ }^{\circ} \mathrm{C}\right)$, and $T_{\text {a.NOCT }}$ is the ambient temperature for the nominal operating cell temperature $\left(20^{\circ} \mathrm{C}\right)$.

The PV system was installed in Seoul, South Korea. The PV system was connected to the electrical system of a building with a grid connected type inverter. Values such as the current, voltage, and produced power measured by the inverter were sent to the monitoring system by the sensor box. And the produced energy was monitored on a personal computer (PC), as displayed in Figure 2. The capacity of the PV system was $1750 \mathrm{~W}$, and the efficiencies of the modules and the inverter at standard test conditions were $15.34 \%$ and $96 \%$, respectively. The generated PV outputs were monitored from August 2018 to July 2019. The actual generated PV system specifications are listed in Table 4.

3.4. Error Metrics. In this study, the mean bias error (MBE), mean absolute error (MAE), root mean square error (RMSE), and NASH-Sutcliffe efficiency (NSE) were employed to evaluate the performance of the model. The MBE, MAE, RMSE, and NSE were calculated using equations (14)-(17), respectively. The verification values of each MBE, MAE, and RMSE were close to zero, which means that the observed values were similar to the predicted values. The

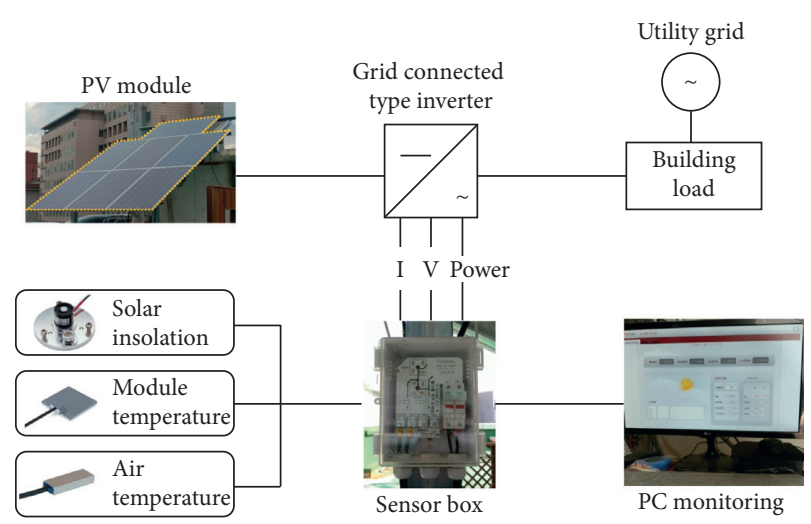

Figure 2: Schematic of the PV and monitoring systems.

TABLE 4: PV system specifications.

\begin{tabular}{lc}
\hline Parameters & Specification \\
\hline Site location & Seoul, South Korea \\
Site longitude & $37^{\circ} 30^{\prime} 12.8^{\prime \prime} \mathrm{N}$ \\
Site latitude & $126^{\circ} 57^{\prime} 25.3^{\prime \prime} \mathrm{E}$ \\
Type of installation & Rooftop installation (elevated) \\
Orientation and tilt & South, $35^{\circ}$ \\
Module type & Multicrystalline silicon \\
Module size & $1640 \times 992 \mathrm{~mm}$ \\
Number of modules & 7 \\
Maximum power & $250 \mathrm{~W}$ \\
Type of PV system & Grid connected \\
Efficiency of module at STC & $15.43 \%$ \\
Efficiency of inverter (EU) & $96 \%$ \\
\hline
\end{tabular}

MBE was used to measure the typical average model deviation. The MBE can provide useful information including whether the predictive model overestimated or underestimated the real values. However, the MBE should be interpreted carefully because positive and negative errors will cancel out. Therefore, the MAE and MBE must be evaluated together. Willmott and Matsuura [43] pointed out that the MAE is a more natural and unambiguous measure of the average error magnitude. The RMSE is often used to determine the success of a prediction model, and it is suitable for determining model precision [44]. If the performance of the prediction model is improved based on the RMSE when evaluating the accuracy of the prediction model, where the RMSE is based on the standard deviation, learning can be used to reduce large errors. The MAE, on the contrary, is sensitive to small errors. Hence, in this study, the RMSE was used during the evaluation of the prediction model to reduce the maximum error of the predicted solar insolation. NSE, on the contrary, is a normalized measure that compares the mean square error estimated by a particular model simulation to the variance of the observed value during the period under investigation [45]. The range of NSE is between 1 and $-\infty$. An NSE value of 1 represents a complete match between the observations and the forecasts, while a smaller NSE value implies that there is less consistency between the measured value and the model value. Additionally, the MAE, MBE, and NSE were evaluated together: 


$$
\begin{aligned}
\mathrm{MBE} & =\frac{1}{n} \sum_{i=1}^{n}(\widehat{H}-H), \\
\mathrm{MAE} & =\frac{1}{n} \sum_{i=1}^{n}|\widehat{H}-H|, \\
\mathrm{RMSE} & =\sqrt{\frac{1}{n} \sum_{i=1}^{n}(\widehat{H}-H)^{2},} \\
\mathrm{NSE} & =1-\frac{\sum(H-\widehat{H})^{2}}{\sum(H-\bar{H})^{2}},
\end{aligned}
$$

where $\widehat{H}, H$, and $\bar{H}$ are the predicted, actual, and average actual solar insolation values, respectively.

The error ranges of the daily prediction models presented in the previous studies were RMSE $2.304-3.624 \mathrm{kWh} /$ $\mathrm{m}^{2} /$ day, MBE $0.120-2.400 \mathrm{kWh} / \mathrm{m}^{2} /$ day, and MAE $1.488-2.592 \mathrm{kWh} / \mathrm{m}^{2} /$ day $[12,46,47]$.

\section{Results and Discussion}

4.1. Prediction Performance of the Solar Insolation Model. The solar insolation on any day can be predicted by the weather data of the previous day. The prediction model used in this research was an MLF. The initial number of hidden neurons for the initial 9 inputs in the hidden layer was determined to be 19 , based on equation (2). In order to increase the accuracy of the prediction model, the number of hidden neurons was extended from 10 to 20 with reference to the initial number of neurons, considering the reduction of inputs. The initial model was predicted using the following initial input valuables: highest and lowest temperature, minimum humidity, precipitation, precipitation duration, wind speed, sunshine duration, continued sunshine duration, and cloud cover. In the second model, precipitation and precipitation duration, which were poor predictors, were removed. Then, the less correlated cloud cover was excluded from the input variables. Cloud cover is the weather factor that has the most significant influence on the solar insolation in the present or near future; however, it has a low effect on the next day's solar insolation.

The accuracy of the predicted model with various NHN and NHL is shown in Table 5. The range of predicted results varied according to the range of input variables. A higher number of input variables does not increase the accuracy of predictions. In particular, when the input variables were reduced from 8 to 7 , the prediction accuracy decreased. However, the prediction accuracy was at its highest when the input variables were further reduced to six highly correlated input variables. This can be attributed to the correlation between the predicted value and the input value and the relationship between the input variables.

The optimal prediction model was that with the smallest RMSE among the 55 prediction result values with 6 input variables, namely, highest and lowest temperature, minimum humidity, wind speed, sunshine duration, and continued sunshine duration. The calculated RMSE values ranged from a minimum of $1.421 \mathrm{kWh} / \mathrm{m}^{2} /$ day to a maximum of $1.720 \mathrm{kWh} / \mathrm{m}^{2} /$ day depending on the number of hidden neurons and hidden layers. The optimal model from the RMSE consisted of 3 hidden layers and 11 hidden neurons. The RMSE, MBE, and MAE of the optimal prediction model were $1.421 \mathrm{kWh} / \mathrm{m}^{2} /$ day, $-0.227 \mathrm{kWh} / \mathrm{m}^{2}$ / day, and $1.133 \mathrm{kWh} / \mathrm{m}^{2} /$ day, respectively. For this model, the average of the errors and the predicted values with large errors were smaller than those of the other models $[11,20,46]$. The RMSE limit presented in this model was lower than the RMSE mentioned in Section 3.4, which is better than the existing model. According to the MBE, the predicted values tended to be underestimated. In Table 6, the optimal prediction model of 3 hidden layers and 11 hidden neurons was compared to the regression model derived in the previous study [21]. Similar results were obtained from the RMSE, MBE, and MAE of the predicted values derived by these two models; however, the NSE values showed better predictive models by the optimal MLF model. This means that the MLF with fewer input variables reflects the daily fluctuation better than the regression model.

Figure 3 presents the daily solar insolation over a year, which was predicted and observed by the meteorological administration. The number of days when the predicted value was lesser than the observed value was 198 in total, and the number of days when the predicted value was higher than the observed value was 159 in total. Generally, the predicted value is lesser than the observed value in spring when the solar insolation increases. This is because the weather data do not reflect the solar insolation increase due to the movement of the Sun and the Earth. The sunshine duration was used to reflect the change in the orbits of the Sun and the Earth. The average solar insolation change pattern was reflected. According to Sun et al [48], a nonlinear approach to a prediction model should be considered due to the nonlinear variation in meteorological variables. However, the daily fluctuation was not reflected fully because a nonlinear approach was not utilized in this model.

With regard to the monthly accuracy of the optimal prediction model (see Table 7), the error from April to September was larger than that of the other months. These months' RMSE and MAE values were greater than the yearly RMSE and MAE. As shown in Table 7, the standard deviation of the measured solar insolation was greater than that of the other months. In particular, from April to September, the solar insolation was abundant, but it varied greatly in response to sudden changes in the weather, such as heavy rainfall. Table 8 and Figure 4 show the range of measured solar insolation in each month. The difference in the maximum and minimum measured insolation for a month was at least $5.7 \mathrm{kWh} / \mathrm{m}^{2} /$ day during the spring and summer seasons. There were 29 days out of 357 days when the difference between the predicted and measured values was over $2.5 \mathrm{kWh} / \mathrm{m}^{2} /$ day. These days occurred between March and October but mostly between May and September. As a result of analyzing the changes in meteorological factors on the day before and days with a large error, the change in continued sunshine duration and average cloud cover was 
TABle 5: Accuracies of predicted value ranges according to the input variables, NHN, and NHL.

\begin{tabular}{|c|c|c|c|c|c|}
\hline Input variables & Number of hidden layers* & $\begin{array}{c}\text { RMSE } \\
\left(\mathrm{kWh} / \mathrm{m}^{2} / \text { day }\right)\end{array}$ & $\begin{array}{c}\mathrm{MBE} \\
\left(\mathrm{kWh} / \mathrm{m}^{2} / \text { day }\right) \\
\end{array}$ & $\begin{array}{c}\text { MAE } \\
\left(\mathrm{kWh} / \mathrm{m}^{2} / \text { day }\right)\end{array}$ & NSE \\
\hline \multirow{5}{*}{$\begin{array}{l}T_{\text {high }}, T_{\text {low }}, \mathrm{RH}, \mathrm{DWS}, \mathrm{PR}, \\
\mathrm{PD}, \mathrm{SD}, \mathrm{CSD}, \mathrm{CC}\end{array}$} & 1 & $1.764-2.000$ & -0.891 to -0.787 & $1.368-1.529$ & -0.503 to -0.169 \\
\hline & 2 & $1.763-2.023$ & -0.811 to -0.649 & $1.355-1.552$ & -0.920 to -0.210 \\
\hline & 3 & $1.760-2.015$ & -0.840 to -0.568 & $1.373-1.506$ & -1.092 to -0.164 \\
\hline & 4 & $1.799-2.015$ & -0.903 to -0.711 & $1.382-1.559$ & -0.937 to -0.216 \\
\hline & 5 & $1.793-2.102$ & -0.853 to -0.589 & $1.366-1.631$ & -1.068 to -0.207 \\
\hline \multirow{5}{*}{$\begin{array}{l}T_{\text {high }}, T_{\text {low }}, \text { RH, DWS, } \\
\text { SD, CSD, CC }\end{array}$} & 1 & $1.975-2.227$ & -1.301 to -0.969 & $1.515-1.692$ & -0.292 to -0.016 \\
\hline & 2 & $1.975-2.138$ & -1.076 to -0.714 & $1.550-1.635$ & -0.162 to -0.016 \\
\hline & 3 & $1.792-2.318$ & -1.221 to -0.567 & $1.432-1.773$ & -0.391 to 0.164 \\
\hline & 4 & $1.889-2.187$ & -1.005 to -0.547 & $1.436-1.632$ & -0.245 to 0.071 \\
\hline & 5 & $1.807-2.338$ & -1.227 to -0.419 & $1.444-1.773$ & -0.423 to 0.150 \\
\hline \multirow{5}{*}{$T_{\text {high }}, T_{\text {low }}, \mathrm{RH}, \mathrm{DWS}, \mathrm{SD}, \mathrm{CSD}$} & 1 & $1.425-1.506$ & -0.272 to -0.129 & $1.157-1.204$ & $0.411-0.473$ \\
\hline & 2 & $1.423-1.597$ & -0.400 to -0.090 & $1.131-1.297$ & $0.337-0.474$ \\
\hline & 3 & $1.421-1.660$ & -0.376 to -0.114 & $1.131-1.342$ & $0.284-0.482$ \\
\hline & 4 & $1.444-1.692$ & -0.464 to -0.121 & $1.148-1.372$ & $0.256-0.458$ \\
\hline & 5 & $1.450-1.720$ & -0.291 to -0.118 & $1.153-1.405$ & -0.338 to 0.454 \\
\hline
\end{tabular}

${ }^{*}$ The NHN for each layer was conducted by changing both to $10-20$ a

found to be largely as a result of rainfall, humidity variations, and changes in suspended matter concentrations. In 24 out of the 29 days, the previous day's rain came to a halt or it rained on the estimation day.

The RMSE and MAE patterns exhibited similar tendencies to the patterns of the monthly solar insolation according to the Sun's trajectory. The RMSE and MAE values were smallest in December during the winter solstice. The RMSE and MAE values then increased with the increase in solar radiation until June. The solar insolation, RMSE, and MAE values decreased after June. The predicted values for the period between October and December, where the MBE values showed positive values, were overestimated. On the contrary, the predicted values for January to March were underestimated compared to the measured values. The predicted values between October and December were overestimated because of the learning effect of the summer data. Conversely, the predicted values between January and March did not reflect the increase in solar insolation because of the influence of the winter data.

4.2. Application of the Optimal Prediction Model to $P V$ Output Estimations. In order to compare the PV production forecast, the energy production of the $1.75 \mathrm{~kW}$ PV system was monitored for 364 days from August 2018 to July 2019. The weather conditions during the monitoring period are listed in Table 9. Conditions were generally clear; however, there were some rainy days due to the effects of typhoons. There was a precipitation period of 97 days in total during the entire monitoring period. During this period, the average daily cloud cover fluctuated greatly. As a result, the variation in solar insolation was larger than that for the other periods. The power generation range for the PV system was $0.1-10.8 \mathrm{kWh} /$ day depending on the weather conditions.

The predicted solar insolation was estimated by using the optimal solar insolation prediction model. The predicted solar insolation was then used to calculate the PV production by using equation (11). To check the validity of the approach, the energy production was calculated from the measured insolation and compared with the actual energy produced by the PV system. Table 10 presents the accuracy results for the energy production estimations. The predicted power generation calculated from the measured insolation was similar to the actual power generation, as shown in Figure 5. There was a difference between the power generation calculated by the measured insolation and the actual production values. Detailed meteorological observations cannot be performed at all PV installation sites. As a result, it is determined that an error occurred due to the positional difference. It is also possible that a difference occurred due to the limitation of the numerical calculations. However, the RMSE, MAE, and MBE of the energy generation calculated from the measured insolation and the actual production were $0.85 \mathrm{kWh} /$ day, $0.38 \mathrm{kWh} /$ day, and $0.66 \mathrm{kWh} /$ day, respectively, indicating that the calculation procedure could be used for estimating the energy production of a PV system. The energy production was calculated using the estimated solar insolation using the proposed procedure. The power generation obtained by the predicted insolation fluctuated less than the actual energy production. Some errors were due to the insufficient predictions of the daily weather condition changes. Although the forecast model reflected the daily variation, it did not predict the actual magnitude of the change. In the spring and summer observation, there was a wide variation in the day to day solar radiation. This was because, unlike previous studies in which the prediction was by hours, the forecast horizon was long and did not reflect all of the variability during that forecast periods. The prediction model proposed in this study predicts the next day's solar insolation by using the weather conditions of the previous day. However, the weather conditions of the monitoring period changed extensively each day, which made it difficult to predict the insolation. The energy production error was especially significant on days when the solar radiation suddenly changed due to rain or snow. In order to provide 
TABLE 6: Comparison of the regression and optimal models of MLF.

\begin{tabular}{|c|c|c|c|c|}
\hline Model & RMSE (kWh/m²/day) & MBE $\left(\mathrm{kWh} / \mathrm{m}^{2} /\right.$ day $)$ & MAE $\left(\mathrm{kWh} / \mathrm{m}^{2} /\right.$ day $)$ & NSE \\
\hline Regression model & 1.428 & -0.074 & 1.179 & -0.771 \\
\hline Optimal model of MLF & 1.421 & -0.227 & 1.133 & 0.482 \\
\hline
\end{tabular}

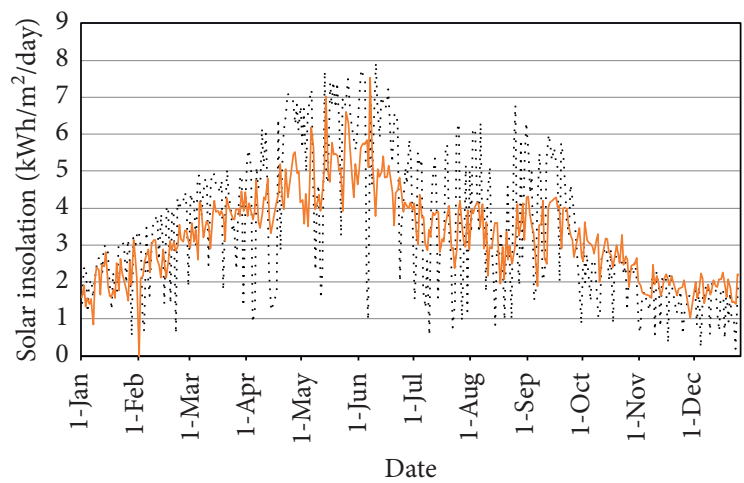

..... Observed value

- Predicted value

(a)

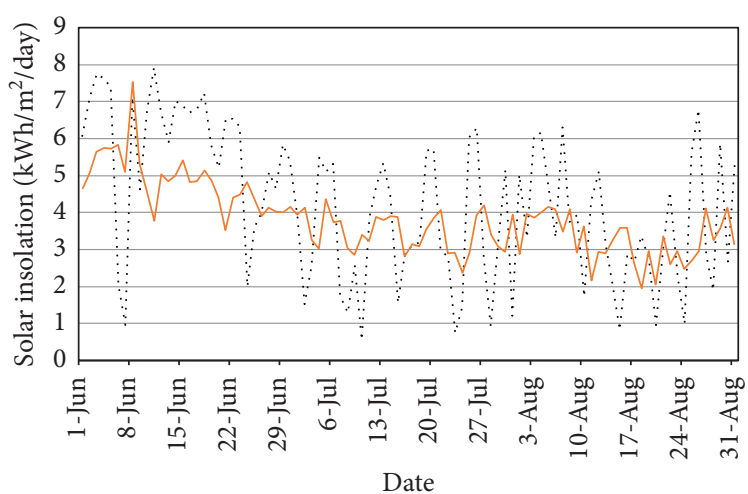

Observed value

_ Predicted value

(c)

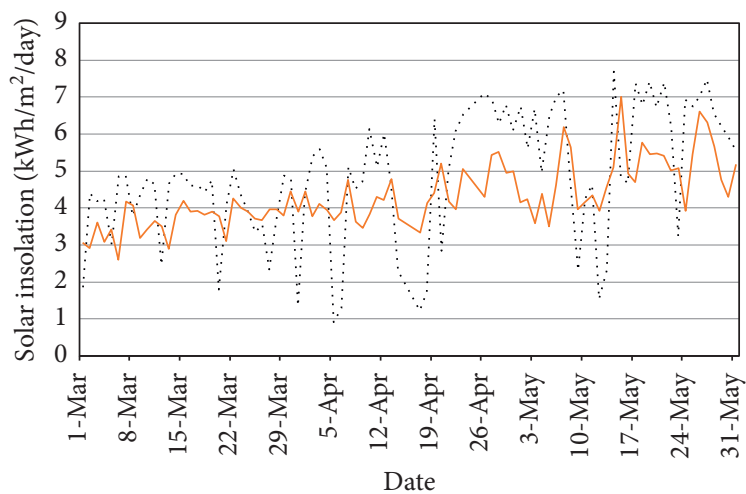

...... Observed value

_ Predicted value

(b)

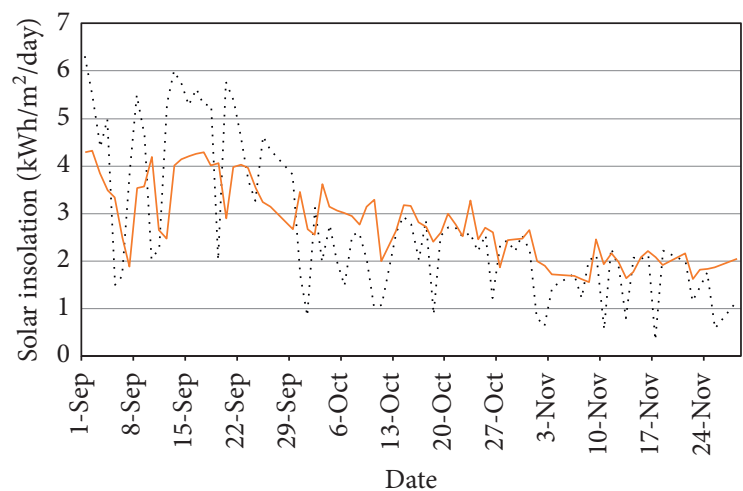

Observed value

_ Predicted value

(d)

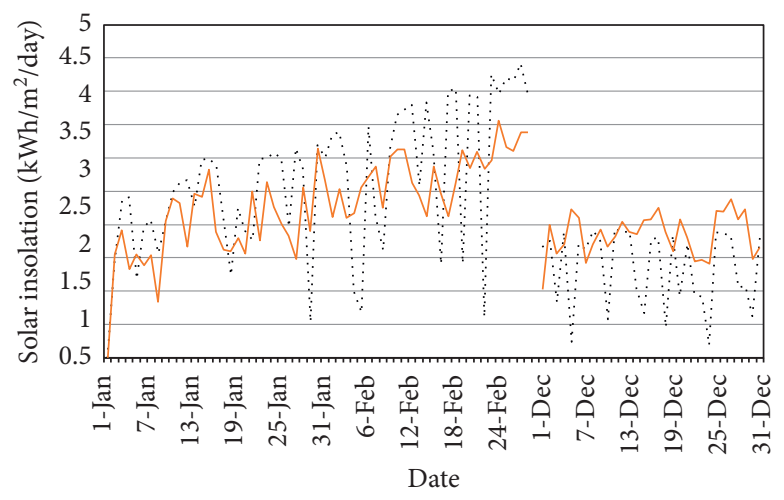

. Observed value

_ Predicted value

(e)

FIGURE 3: Comparison between the predicted and observed values of daily solar insolation: (a) over one year; (b) spring; (c) summer; (d) fall; (e) winter. 
TABLE 7: Accuracies of the optimal predicted values as compared to the measured values.

\begin{tabular}{lcccc}
\hline Month & Number of valid days & RMSE $\left(\mathrm{kWh} / \mathrm{m}^{2} /\right.$ day $)$ & $\mathrm{MBE}\left(\mathrm{kWh} / \mathrm{m}^{2} /\right.$ day $)$ & $\mathrm{MAE}\left(\mathrm{kWh} / \mathrm{m}^{2} / \mathrm{day}\right)$ \\
\hline January & 31 & 0.717 & -0.376 & 0.580 \\
February & 28 & 1.133 & -0.246 & 1.003 \\
March & 31 & 1.112 & -0.356 & 0.917 \\
April & 28 & 1.742 & -0.595 & 1.581 \\
May & 31 & 1.753 & -0.797 & 1.536 \\
June & 30 & 2.034 & -0.901 & 1.744 \\
July & 31 & 1.760 & 0.029 & 1.485 \\
August & 31 & 1.753 & -0.439 & 1.491 \\
September & 29 & 1.650 & -0.432 & 0.559 \\
October & 30 & 0.925 & 0.609 & 0.643 \\
November & 26 & 0.668 & 0.521 & 0.481 \\
December & 31 & 0.767 & & 0.595 \\
\hline
\end{tabular}

TABLE 8: Range of measured solar insolation (unit: $\mathrm{kWh} / \mathrm{m}^{2} /$ day).

\begin{tabular}{lccccc}
\hline Month & Maximum value & Minimum value & Mean value & Difference between maximum and minimum values & Standard deviation \\
\hline January & 3.34 & 0.54 & 2.37 & 2.80 & 0.70 \\
February & 4.42 & 0.61 & 3.04 & 3.80 & 1.20 \\
March & 5.05 & 1.35 & 4.07 & 6.70 & 0.97 \\
April & 7.10 & 0.91 & 4.94 & 6.15 & 1.87 \\
May & 7.70 & 1.55 & 5.77 & 6.97 & 1.65 \\
June & 7.93 & 0.96 & 5.72 & 5.73 & 1.81 \\
July & 6.29 & 0.56 & 3.42 & 5.96 & 1.76 \\
August & 6.79 & 0.83 & 3.73 & 6.00 & 1.74 \\
September & 6.00 & 0.01 & 3.95 & 2.49 & 0.70 \\
October & 3.11 & 0.62 & 2.14 & 1.96 & 0.60 \\
November & 2.31 & 0.34 & 1.59 & 1.75 & 0.58 \\
December & 1.95 & 0.19 & 1.36 & &
\end{tabular}

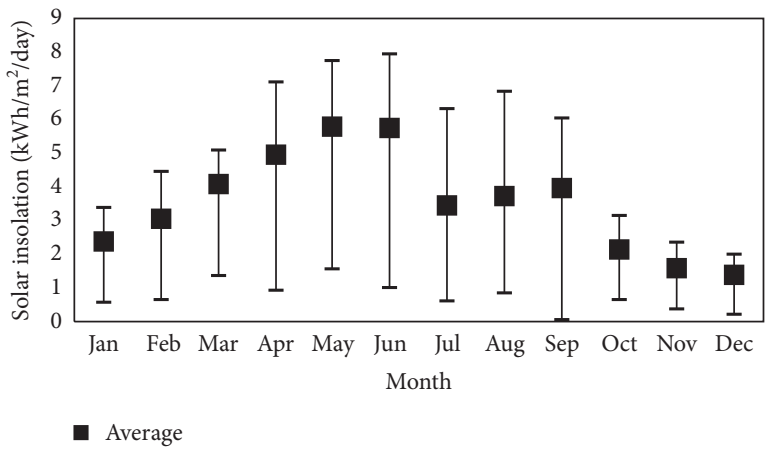

FIGURE 4: Range of monthly solar insolation by measured data from the meteorological administration.

accurate forecasts of the energy production according to weather condition changes as basic information for energy trading, it will be necessary to compensate for dramatic fluctuations in weather conditions. The error magnitude was smaller in the fall and winter periods with less precipitation, but these seasons were predicted to be less than the actual energy production. In regions with distinct characteristics of seasons, it is necessary to present the solar insolation prediction model by season.

P2P energy trading should also take into account the energy price and the energy consumption of buildings
TABle 9: Weather conditions during the monitoring period.

\begin{tabular}{lc}
\hline Weather elements & Value \\
\hline $\begin{array}{l}\text { Monitoring period } \\
\text { The day's highest }\end{array}$ & 2 August 2018 to 31 July $2019(364$ \\
temperature & $-6.6-39.6^{\circ} \mathrm{C}$ \\
Minimum humidity & $10-91 \%$ \\
Daily wind speed & $0.8-3.8 \mathrm{~m} / \mathrm{s}$ \\
Number of rainy days & 97 days \\
Sunshine duration & $9.6-14.8$ hours \\
\hline
\end{tabular}

according to weather conditions and operating schedules. In general, the summer and winter energy consumption in buildings is related to the external weather conditions. On days where energy consumption is expected to be high in buildings, the energy produced by PV systems will be consumed preferentially in buildings, even if less or more energy is produced through PV systems. On the contrary, if the prosumer is planning to sell the produced energy to the grid because the projected energy consumption is low in the building, the energy trade profits may be less than expected if the system actually produces less power than expected. In order to propose the optimal operations for P2P energy trading, it is necessary to predict the energy consumption of the building, the price of energy, and the amount of energy 
TABLE 10: Accuracies of the energy production estimations.

\begin{tabular}{lccc}
\hline & RMSE (kWh/day) & MBE (kWh/day) & MAE (kWh/day) \\
\hline Energy production by measured insolation & 0.85 & 0.38 & 0.66 \\
Energy production by predicted insolation & & & -0.36 \\
$\quad$ Year & 2.70 & 0.05 & 2.28 \\
Spring (Mar, Apr, and May) & 3.25 & 0.42 & 2.60 \\
Summer (Jun, Jul, and Aug) & 2.57 & -0.08 & 2.17 \\
Fall (Sep, Oct, and Nov) & 2.43 & -1.13 & 2.14 \\
Winter (Dec, Jan, and Feb) & 2.45 & & 2.20 \\
\hline
\end{tabular}

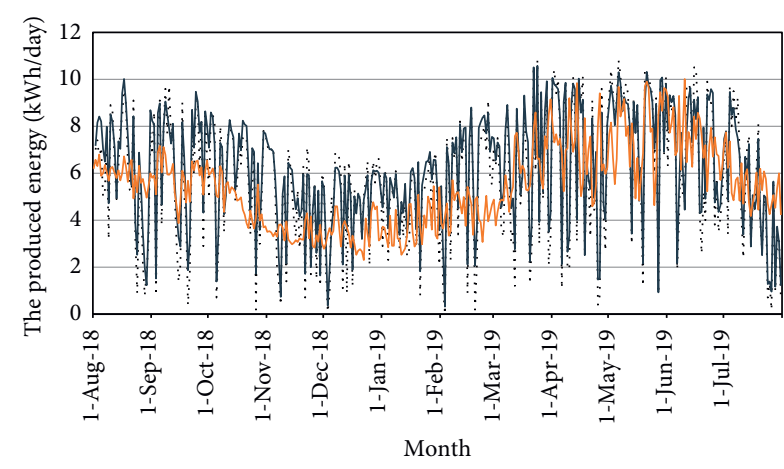

Monitored power generation by the PV system

__ Estimated power generation by predicted irradiance

_ Estimated power generation by measured irradiance

(a)

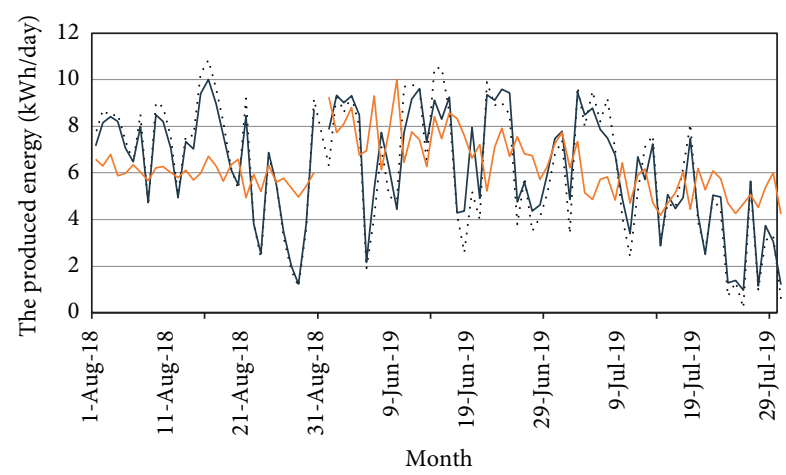

Monitored power generation by the PV system

- Estimated power generation by predicted irradiance

_ Estimated power generation by measured irradiance

(c)

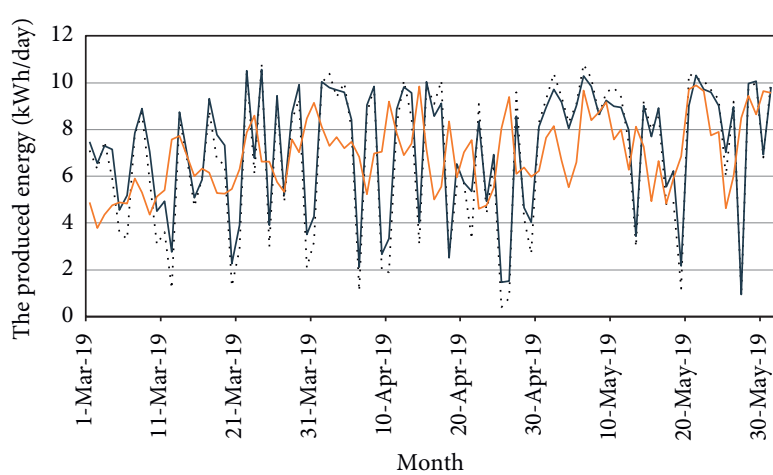

..... Monitored power generation by the PV system

__ Estimated power generation by predicted irradiance

__ Estimated power generation by measured irradiance

(b)

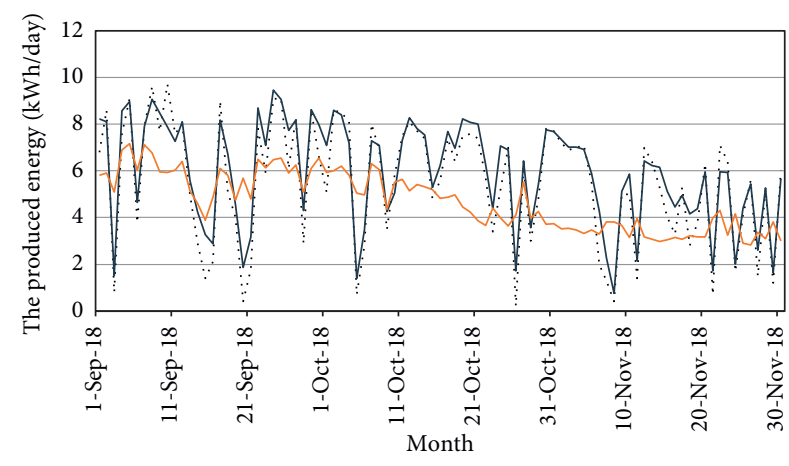

Monitored power generation by the PV system

Estimated power generation by predicted irradiance

Estimated power generation by measured irradiance

(d)

FIgURE 5: Continued. 


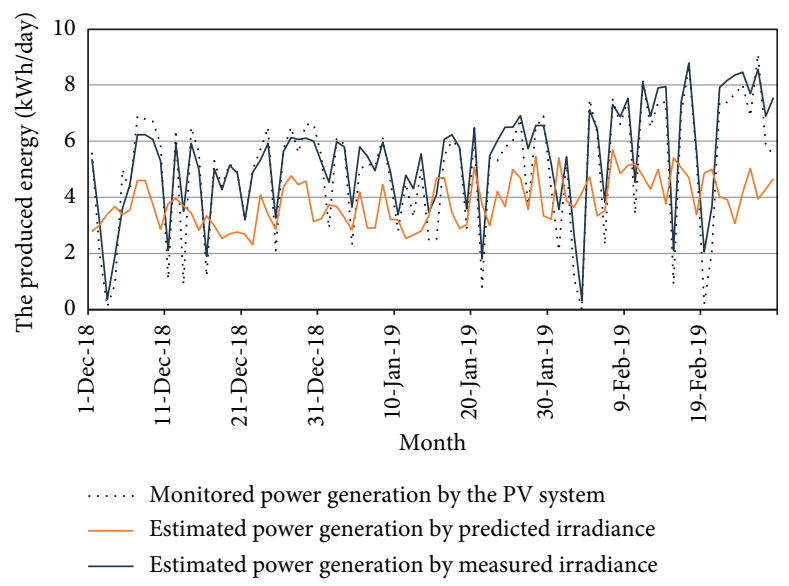

(e)

FIGURE 5: Comparison of the predicted and measured energy production insolations: (a) over one year; (b) spring; (c) summer; (d) fall; (e) winter.

to be produced by PV systems together to suggest the optimal use method.

\section{Conclusions}

The recent energy-saving building policies enacted in South Korea are expected to lead to the realization of distributed energy generation in a smart grid. P2P trading of the energy produced in buildings will soon take place. Predicting energy production is essential for $\mathrm{P} 2 \mathrm{P}$ energy trading planning, and the energy produced through PV systems will be mainly traded. In this study, the potential applicability of the energy production prediction of PV systems was investigated by using the predicted solar insolation derived from a simple model to provide information to prosumers engaging in small-scale electricity trading. The predictive model proposed by the existing researchers attempted to accurately predict the hourly or daily radiation by using a large amount of information. However, in this study, the amount of solar radiation the next day was predicted by using a small amount of weather information. The results are summarized as follows:

(1) The prediction model for solar insolation was built by using an MLF, and the proposed model uses the daily weather conditions. The predicted solar insolation was based on the estimated weather conditions on the day before the prediction. To find the optimal architecture, the number of input variables, the number of hidden neurons, and the number of hidden layers were changed.

(2) The input variables of the optimal model included the highest and lowest temperature, minimum humidity, wind speed, sunshine duration, and continued sunshine duration. The optimal model consisted of 3 hidden layers and 11 hidden neurons.

(3) The energy output of the PV system was calculated from the solar insolation estimated by the optimal model. The calculated energy output was compared to the actual PV system's output. The accuracy indexes for the PV system's energy output using the optimal model, which included the root mean squared error, mean bias error, and mean absolute error, were $2.70 \mathrm{kWh} / \mathrm{m}^{2} /$ day, $-0.36 \mathrm{kWh} / \mathrm{m}^{2} /$ day, and $2.28 \mathrm{kWh} / \mathrm{m}^{2} /$ day, respectively.

The error of the predicted values for solar insolation varied from season to season. For instance, in the summer when the difference between the maximum and minimum insolation was large, results did not fully reflect insolation changes in response to weather changes. The predicted values tended to represent the median value. Although this provides stable values, data were less predictable in the summer when energy production was high. On the contrary, in winter, the accuracy of the forecasted insolation was high for high solar insolation days. However, the predictions were higher than the actual solar insolation for the low solar insolation days. The proposed model did not reflect sudden weather changes on any day because it used the daily weather conditions of the day before the forecast. To further improve the prediction model, it is necessary to execute the prediction model in the morning or on an hourly basis rather than the day before the forecast and to further optimize the prediction model in accordance with the variations in seasonal characteristics.

The prediction of the PV system energy production through solar insolation predictions showed a pattern similar to the solar insolation prediction results. The energy production estimation by the predicted insolation tended to yield underestimates compared to the real produced energy. The monitoring period was characterized by severe weather fluctuations. To accommodate such weather fluctuations in the future, it may be necessary to compensate for climate change. The accuracy of the prediction model presented in this study is much lower on sunny days or cloudy days with precipitation. There was a limit to predicting the exact amount of solar radiation, including various weather variables, in the relationship between the weather conditions of the previous day and the weather conditions of the next day. There was no uncertainty about this change in weather. In this research, the 
solar insolation of the horizontal plane was predicted, while that of the inclined insolation was calculated by the equations and added to the solar power generation equation. In this process, there was a difference between the actual value and the predicted value obtained using the equation. In the future, it may be necessary to distinguish the prediction model results according to weather conditions or to increase the accuracy by shortening the prediction horizon.

If P2P energy trading becomes active in the future, it will be necessary to predict the energy production based on the weather conditions of the forecast day. Therefore, an accurate insolation prediction model will be needed to predict the energy production of the PV systems involved in P2P energy trading. The model described here represents a good potential model for such purposes, and future optimization work will be forthcoming.

\section{Data Availability}

The data used to support the findings of this study are available from the corresponding author upon request.

\section{Conflicts of Interest}

The author declares that there are no conflicts of interest.

\section{Acknowledgments}

This research was supported by the Basic Science Research Program through the National Research Foundation of Korea (NRF), funded by the Ministry of Science, ICT \& Future Planning (NRF-2017R1C1B20117911).

\section{References}

[1] Korea Energy Economic Institute, Energy Consumption Survey, Ministry of Trade, Industry and Energy, Sejong City, South Korea, 2015.

[2] International Energy Agency, Key World Energy Statistics, International Energy Agency, Paris, France, 2017.

[3] I. S. Bayram, M. Z. Shakir, M. Abdallah, and K. Qaraqe, "A survey on energy trading in smart grid," in Proceedings of the 2014 IEEE Global Conference on Signal and Information Processing (GlobalSIP), pp. 258-262, IEEE, Atlanta, GA, USA, December 2014.

[4] S.-V. Oprea, A. Bâra, A. Uţă, A. Pîrjan, and G. Căruţaşu, "Analyses of distributed generation and storage effect on the electricity consumption curve in the smart grid context," Sustainability, vol. 10, no. 7, p. 2264, 2018.

[5] E. Mengelkamp, J. Gärttner, K. Rock, S. Kessler, L. Orsini, and C. Weinhardt, "Designing microgrid energy markets," $A p$ plied Energy, vol. 210, pp. 870-880, 2018.

[6] C. Park and T. Yong, "Comparative review and discussion on P2P electricity trading," Energy Procedia, vol. 128, no. 3-9, 2017.

[7] C. Zhang, J. Wu, C. Long, and M. Cheng, "Review of existing peer-to-peer energy trading projects," Energy Procedia, vol. 105, pp. 2563-2568, 2017.

[8] J. Jimeno, J. Anduaga, J. Oyarzabal, and A. G. de Muro, "Architecture of a microgrid energy management system," European Transactions on Electrical Power, vol. 21, no. 2, pp. 1142-1158, 2011.
[9] K. Alanne and A. Saari, "Distributed energy generation and sustainable development," Renewable and Sustainable Energy Reviews, vol. 10, no. 6, pp. 539-558, 2006.

[10] J. Thomsen, N. S. Hussein, C. Senkpiel, N. Hartmann, and T. Schlegl, "An optimized energy system planning and operation on distribution grid level-the decentralized market agent as a novel approach," Sustainable Energy, Grids and Networks, vol. 12, pp. 40-56, 2017.

[11] J. Soares, A. P. Oliveira, M. Z. Božnar, P. Mlakar, J. F. Escobedo, and A. J. Machado, "Modeling hourly diffuse solar-radiation in the city of São Paulo using a neural-network technique," Applied Energy, vol. 79, no. 2, pp. 201-214, 2004.

[12] P. Mathiesen and J. Kleissl, "Evaluation of numerical weather prediction for intra-day solar forecasting in the continental United States," Solar Energy, vol. 85, no. 5, pp. 967-977, 2011.

[13] H. Sun, D. Gui, B. Yan et al., "Assessing the potential of random forest method for estimating solar radiation using air pollution index," Energy Conversion and Management, vol. 119, pp. 121-129, 2016.

[14] A. Sharma and A. Kakkar, "Forecasting daily global solar irradiance generation using machine learning," Renewable and Sustainable Energy Reviews, vol. 82, no. 3, pp. 2254-2269, 2018.

[15] F.-V. Gutierrez-Corea, M.-A. Manso-Callejo, M.-P. MorenoRegidor, and M.-T. Manrique-Sancho, "Forecasting shortterm solar irradiance based on artificial neural networks and data from neighboring meteorological stations," Solar Energy, vol. 134, pp. 119-131, 2016.

[16] J. Huang and R. J. Davy, "Predicting intra-hour variability of solar irradiance using hourly local weather forecasts," Solar Energy, vol. 139, pp. 633-639, 2016.

[17] M. Vakili, S. R. Sabbagh-Yazdi, S. Khosrojerdi, and K. Kalhor, "Evaluating the effect of particulate matter pollution on estimation of daily global solar radiation using artificial neural network modeling based on meteorological data," Journal of Cleaner Production, vol. 141, pp. 1275-1285, 2017.

[18] X. Qing and Y. Niu, "Hourly day-ahead solar irradiance prediction using weather forecasts by LSTM," Energy, vol. 148, pp. 461-468, 2018.

[19] B. Amrouche and X. Le Pivert, "Artificial neural network based daily local forecasting for global solar radiation," Applied Energy, vol. 130, pp. 333-341, 2014.

[20] H. Long, Z. Zhang, and Y. Su, "Analysis of daily solar power prediction with data-driven approaches," Applied Energy, vol. 126, pp. 29-37, 2014.

[21] M. H. Chung, "Modelling of solar irradiance forecasting using local meteorological data," KIEAE Journal, vol. 17, no. 6, pp. 273-278, 2017.

[22] M. H. Chung, "The correlation of solar radiation and atmospheric elements including air pollution," KIEAE Journal, vol. 19, no. 1, pp. 69-74, 2019.

[23] J. Sola and J. Sevilla, "Importance of input data normalization for the application of neural networks to complex industrial problems," IEEE Transactions on Nuclear Science, vol. 44, no. 3, pp. 1464-1468, 1997.

[24] C. F. M. Coimbra, H. T. C. Pedro, and J. Kleissl, "Stochasticlearning methods," in Solar Energy Forecasting and Resource Assessment, pp. 383-406, Academic Press, Oxford, UK, 1st edition, 2013.

[25] E. W. Law, A. A. Prasad, M. Kay, and R. A. Taylor, "Direct normal irradiance forecasting and its application to concentrated solar thermal output forecasting-a review," Solar Energy, vol. 108, pp. 287-307, 2014. 
[26] M. Daigne, M. David, P. Lauret, J. Boland, and N. Schmutz, "Review of solar irradiance forecasting methods and a proposition for small-scale insular grids," Renewable and Sustainable Energy Reviews, vol. 27, pp. 65-76, 2013.

[27] A. Mellit and A. M. Pavan, "A 24-h forecast of solar irradiance using artificial neural network: application for performance prediction of a grid-connected PV plant at Trieste, Italy," Solar Energy, vol. 84, no. 5, pp. 807-821, 2010.

[28] P. Lauret, E. Fock, R. N. Randrianarivony, and J.-F. ManicomRamsamy, "Bayesian neural network approach to short time load forecasting," Energy Conversion and Management, vol. 49, no. 5, pp. 1156-1166, 2008.

[29] R. Marquez and C. F. M. Coimbra, "Forecasting of global and direct solar irradiance using stochastic learning methods, ground experiments and the NWS database," Solar Energy, vol. 85, no. 5, pp. 746-756, 2011.

[30] F. S. Tymvios, C. P. Jacovides, S. C. Michaelides, and C. Scouteli, “Comparative study of Ångström's and artificial neural networks' methodologies in estimating global solar radiation," Solar Energy, vol. 78, no. 6, pp. 752-762, 2005.

[31] S. Haykin, Neural Networks-A Comprehensive Foundation, Pearson Prentice Hall, New York, USA, 3rd edition, 2008.

[32] J. Yang, H. Rivard, and R. Zmeureanu, "On-line building energy prediction using adaptive artificial neural networks," Energy and Buildings, vol. 37, no. 12, pp. 1250-1259, 2005.

[33] J.-L. Zhang, "On the convergence properties of the LevenbergMarquardt method," Optimization, vol. 52, no. 6, pp. 739756, 2003.

[34] L. S. H. Ngia and J. Sjoberg, "Efficient training of neural nets for nonlinear adaptive filtering using a recursive LevenbergMarquardt algorithm," IEEE Transactions on Signal Processing, vol. 48, no. 7, pp. 1915-1927, 2000.

[35] F. M. Dias, A. Antunes, J. Vieira, and A. M. Mota, "On-line training of neural networks: a sliding window approach for the levenberg-marquardt algorithm," in Proceedings of the International Work-Conference on the Interplay between Natural and Artificial Computation, pp. 577-585, Springer, Las Palmas, Spain, June 2005.

[36] J. W. Moon, "Performance of ANN-based predictive and adaptive thermal-control methods for disturbances in and around residential buildings," Building and Environment, vol. 48, pp. 15-26, 2012.

[37] B. Liu and R. Jordan, "Daily insolation on surfaces tilted towards equator," ASHRAE Transactions, vol. 67, pp. 526-541, 1962.

[38] J. K. Page, “The estimation of monthly mean values of daily total short wave radiation on vertical and inclined surface from sunshine records for latitude $40^{\circ} \mathrm{N}-40^{\circ} \mathrm{S}$," in Proceedings of the UN Conference on New Sources of Energy, vol. 4, pp. 378-390, Rome, Italy, August 1961.

[39] J. V. Paatero and P. D. Lund, "Effects of large-scale photovoltaic power integration on electricity distribution networks," Renewable Energy, vol. 32, no. 2, pp. 216-234, 2007.

[40] M. E. Ropp, M. Begovic, and A. Rohatgi, "Determination of the curvature derating factor for the georgia tech aquatic center photovoltaic array," in Proceedings of the Conference Record of the Twenty-Sixth IEEE Photovoltaic Specialists Conference, pp. 1297-1300, Anaheim, CA, USA, September 1997.

[41] Ö. Ayvazoğluyüksel and Ü. B. Filik, "Estimation methods of global solar radiation, cell temperature and solar power forecasting: A review and case study in Eskişehir," Renewable and Sustainable Energy Reviews, vol. 91, pp. 639-653, 2018.
[42] W. Omran, Performance Analysis of Grid-Connected Photovoltaic System, Ph.D. dissertation, Department of Electrical and Computer Engineering, University of Waterloo, Ontario, Canada, 2010.

[43] C. J. Willmott and K. Matsuura, "Advantages of the mean absolute error (MAE) over the root mean square error (RMSE) in assessing average model performance," Climate Research, vol. 30, no. 1, pp. 79-82, 2009.

[44] T. Chai and R. R. Draxler, "Root mean square error (RMSE) or mean absolute error (MAE)?-arguments against avoiding RMSE in the literature," Geoscientific Model Development, vol. 7, no. 3, pp. 1247-1250, 2014.

[45] J. E. Nash and J. V. Sutcliffe, "River flow forecasting through conceptual models part I - A discussion of principles," Journal of Hydrology, vol. 10, no. 3, pp. 282-290, 1970.

[46] Y. Chu, H. T. C. Pedro, M. Li, and C. F. M. Coimbra, "Realtime forecasting of solar irradiance ramps with smart image processing," Solar Energy, vol. 114, pp. 91-104, 2015.

[47] C. Cornaro, M. Pierro, and F. Bucci, "Master optimization process based on neural networks ensemble for 24-h solar irradiance forecast," Solar Energy, vol. 111, pp. 297-312, 2015.

[48] H. Sun, N. Zhao, X. Zeng, and D. Yan, "Study of solar radiation prediction and modeling of relationships between solar radiation and meteorological variables," Energy Conversion and Management, vol. 105, pp. 880-890, 2015. 